\title{
Enhancement of FSO Communications Links Under Complex Environment
}

\author{
Satea H. ALNAJJAR*, Ammar A. NOORI ${ }^{*}$, and Arwa A. MOOSA* \\ Department of Network Engineering, Al-iraqia University, Baghdad, Iraq \\ *Corresponding author: Satea H. ALNAJJAR \\ E-mail: dr.satea@gmail.com \\ Ammar A. NOORI \\ E-mail: eng.ammar.noori@gmail.com \\ Arwa A. MOOSA \\ E-mail: eng.arwa.amir@gmail.com
}

\begin{abstract}
Free space optical communication is a line-of-sight (LOS) technology that uses lasers to provide optical bandwidth connections. Potential disturbance arising from the weather condition is one of the most effective factors that influence the bi-directional free space optics (FSO) performance. The complex weather condition in the Middle East region and Arabian Gulf has been dominated by dust storms activities. Dust storms directly affect the characteristics of FSO and consequently lead to an increase in the bit error rate (BER) and deterioration $Q$-factor to bad levels due to the high attenuation factor. In this research, the authors compare the differences between two bi-directional FSOs. One is the traditional link, and the other has been developed to enhance the system performance under the dust storms condition. The proposed design consists of dual FSO channels, and each one includes erbium-doped fiber amplifier (EDFA) optical amplifiers. This design has demonstrated the proficiency in addressing the attenuation that occurs due to weather stickers. The results prove there is an improvement in performance by measuring the $Q$-factor. In addition, BER can be significantly improved, and further communicating distance can be achieved by utilizing $1550 \mathrm{~nm}$ with multiple channels and EDFA.
\end{abstract}

Keywords: FSO; atmospheric attenuation; laser; dust attenuation; EDFA

Citation: Satea H. ALNAJJAR, Ammar A. NOORI, and Arwa A. MOOSA, "Enhancement of FSO Communications Links Under Complex Environment," Photonic Sensors, 2017, 7(2): 113-122.

\section{Introduction}

The weather condition is one of the most effective factors that have a big impact on the free space optics (FSO) link performance. Link range, the bit error rate (BER), quality factor, and optical power will experience fatal degradation in the complex weather environment. Atmospheric attenuation of the FSO system can be triggered by weather fluctuation such as fog, dust storm, snow, rain, and haze since they are the most dominant outage sources in the FSO system [1]. It can affect the system by means of absorption and scattering of laser photons by variety of aerosols and gaseous molecules in the atmosphere. Absorption occurs due to reciprocal action between the photons of the optical signal and the molecules. Scattering also has the same interaction but without affecting the wave length [2]. Studies have shown that optical attenuation can go up to $340 \mathrm{~dB} / \mathrm{km}$ in fog with a visibility of $50 \mathrm{~m}$ [1]. Different studies of FSO link availability and parameters optimization of the transmission link were proposed in the past for

Received: 5 September 2016 / Revised: 30 November 2016

(C) The Author(s) 2017. This article is published with open access at Springerlink.com

DOI: 10.1007/s13320-017-0336-1

Article type: Regular 
various application scenarios. The dedicated indoor laboratory atmospheric chamber to investigate the effects of fog and dust storm on the FSO link performance was developed in [3]. A new laboratory structure to replicate the sandstorm based on fluid dynamics simulations has been addressed and reported in [4]. The optical amplifying-and-forward $(\mathrm{OAF})$ relaying technique using the optical amplifier was first proposed in [5], and in [6] the problem of beam divergence caused by atmospheric turbulence through the implementation of FSO system over atmosphere for a long distance was avoided by using wave length division multiplexing (WDM) and parameters tuning of FSO link to increase the overall system performance.

In this paper, a new FSO link design has been proposed to enhance the system performance under the dust storms condition, extend the link range, and calculate the attenuation caused by dust storms using the Kim model for the laser beam propagation with an approximate visibility of $70 \mathrm{~m}[1,4]$. The new design upgraded by using two FSO channels with erbium-doped fiber amplifier (EDFA) optical amplifiers at the sending and receiving ends to ensure a low BER with an extended distance of up to $2.5 \mathrm{~km}$.

The rest of this paper is organized as follows: Section 2 presents the dust storms attenuation for the FSO and the application of the Kim model to approximate the atmospheric attenuation, Section 3 devotes to the description of the proposed FSO design with a performance comparison among one FSO link channel, two FSO link channels, and two channels with EDFAs, Section 4 shows the simulation results and discussion, and Section 5 is the research conclusions.

\section{Dust storms attenuation for FSO link}

One of the challenges of the FSO channel in which the signal loss and link failure are caused is the atmospheric attenuation due to absorption, scattering, and scintillation [1, 7]. All these effects vary with time and depend on the current local conditions, weather, and distance. Water particles and carbon dioxide mainly cause the absorption of optical signals, whereas the fog, rain, snow, and cloud cause the scattering of optical signals transmitting in the free space. This scattering causes portion of the light beam travelling from a source to deflect away from the intended receiver [1], and the atmospheric attenuation is given by the following Beer's law equation, which represents the relationship between the power of the transmitted signal and the received signal in the presence of the atmospheric attenuation $[1,7]$ :

$$
\tau=P_{L}=P_{0} \times \operatorname{EXP}(-\alpha \times L)
$$

where $P_{L}$ is the laser power at the range $L, P_{0}$ is the laser power of the source, and $\alpha$ is the atmospheric attenuation coefficient. The atmospheric attenuation $(\tau)$ in $\mathrm{dB}$ can be calculated as follows:

$$
\tau_{R}=4.3429 \times \alpha \times L[\mathrm{~dB}]
$$

and the atmospheric attenuation coefficient according to the Kim model equals to

$$
\alpha=\frac{3.91}{V} \times\left(\frac{550 \mathrm{~nm}}{\lambda}\right)^{-q}
$$

where $\lambda$ is the wavelength in $\mathrm{nm}, V$ is the visibility, and $q$ is the size distribution of the scattering particles. The attenuation of the transmitted signal can be obtained from the previous model to various weather conditions using $q[1,6,7]$ :

$$
\begin{aligned}
& q=1.6 \text { for visibility }(V>50 \mathrm{~km}) \\
& q=1.3 \text { for visibility }(6 \mathrm{~km}<V<50 \mathrm{~km}) \\
& q=0.16 V+0.34 \text { for visibility }(1 \mathrm{~km}<V<6 \mathrm{~km}) \\
& q=V-0.5 \text { for visibility }(0.5 \mathrm{~km}<V<1 \mathrm{~km}) \\
& q=0 \text { for visibility }(V<0.5 \mathrm{~km}) .
\end{aligned}
$$

This form of calculation is very handy because for a given wavelength, the amount of the attenuation only depends on the visibility [1]. The visibility is used in the equation above as in $[1,4]$. 
In this research, three different wavelengths are adopted to compare the attenuation effect on each one of them, and the calculations of the atmospheric attenuation coefficient are shown in Table 1.

Table $1 \mathrm{FSO}$ link attenuation in $\mathrm{dB} / \mathrm{km}$ according to the Kim model.

\begin{tabular}{ccccc}
\hline $\begin{array}{c}\text { Visibility } \\
(\mathrm{km})\end{array}$ & $\begin{array}{c}\mathrm{dB} / \mathrm{km} \\
(450 \mathrm{~nm})\end{array}$ & $\begin{array}{c}\mathrm{dB} / \mathrm{km} \\
(785 \mathrm{~nm})\end{array}$ & $\begin{array}{c}\mathrm{dB} / \mathrm{km} \\
(1550 \mathrm{~nm})\end{array}$ & Weather \\
\hline 0.05 & 340 & 340 & 340 & Heavy fog \\
0.07 & 242 & 242 & 242 & Heavy dust \\
0.2 & 85 & 85 & 85 & Moderate \\
0.5 & 34 & 34 & 34 & Dust-fog \\
1 & 19 & 14 & 10 & Rain \\
2 & 10 & 7 & 4 & Haze \\
4 & 5 & 3 & 2 & \\
10 & 2 & 1 & 0.4 & Clear \\
23 & 1 & 0.5 & 0.2 & \\
\hline
\end{tabular}

\section{Design of the proposed FSO system}

FSO is a line-of-sight technology that uses lasers to provide optical bandwidth connections, and it is also an optical communications technique that propagates the light in the free space such as air, outer space, vacuum, or something similar to wirelessly transmit data for telecommunications and computer networking. In recent years, the demand for FSO communications has increased considerably due to its ability to carry high bandwidth data in the long distance up to $8 \mathrm{~km}$ with low bit error rates $[3,4]$.

Figure 1 shows the essential concepts and the devices that are used to design the FSO system utilizing the optical communications system design software "Optisystem" TM" [8].

The receiver component of the FSO on both sides has a specific minimum sensitivity at a given data rate, and the received power must remain above the lowest sensitivity to guarantee the reliable operation of the system. The connectivity of the FSO channel is affected by the carrier medium (air) between the sender and the receiver, thus, the weather conditions impact the signal-transmission process. Therefore, the weather conditions must be considered when using the FSO system. Thus, the system is analyzed under a range of weather conditions, depending on the attenuation coefficient affecting the channel transmitter.

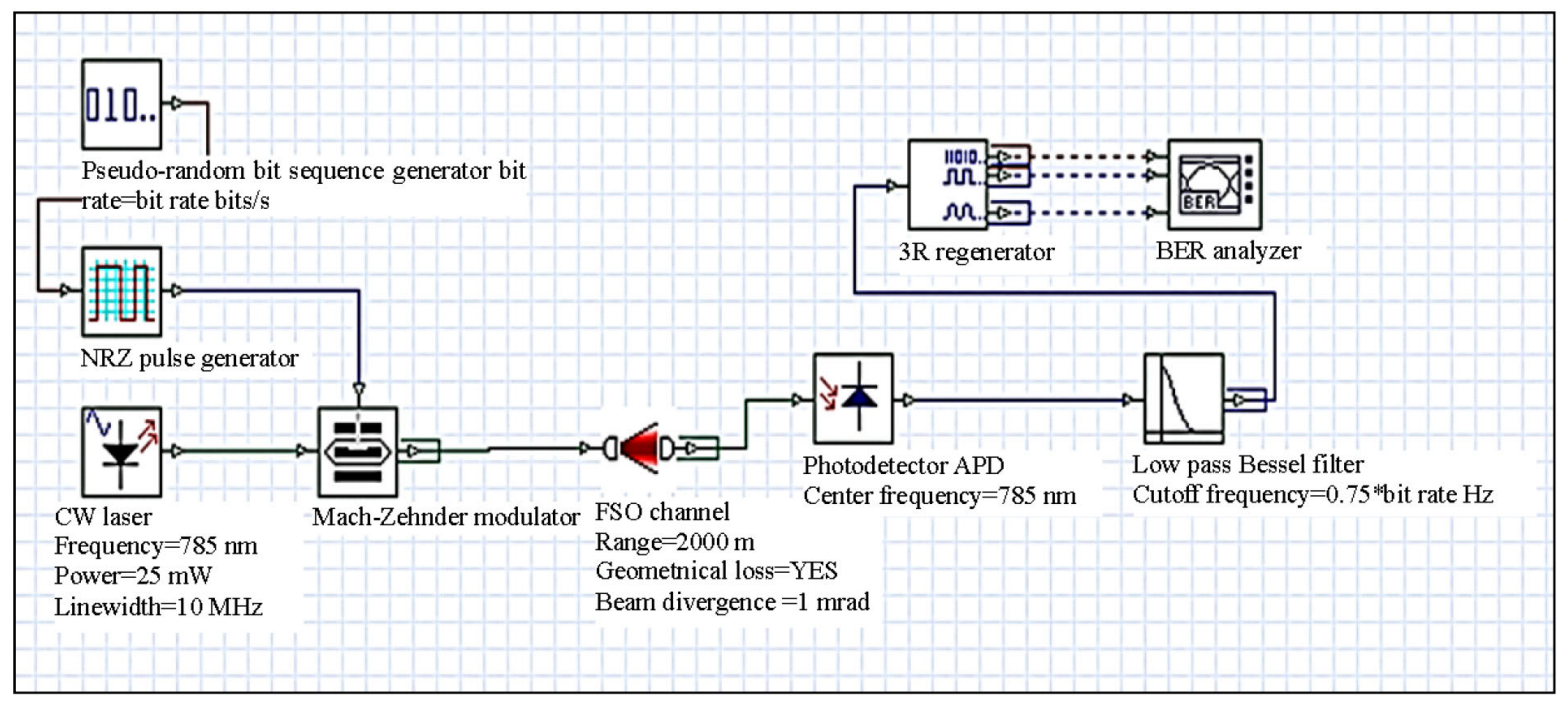

Fig. 1 One-channel FSO system components in "Optisystem" TM".

The FSO transmission part in the system includes a pseudo-random bit generator, a non-return-to-zero (NRZ) pulse generator, continuous wave $(\mathrm{CW})$ laser diodes where it acts as the carrier source with the wavelength $(450 \mathrm{~nm}$, $750 \mathrm{~nm}$, and $1550 \mathrm{~nm}$ ) and a Mach-Zehnder modulator. An avalanche photo diode (APD) and a low-pass Gaussian filter are used in the receiving 
side, which causes $40 \%$ timing jitter reduction and reduces the switch window loss of the traveling-wave electron absorption modulator (TW-EAM) [9].

In this research, the performance comparison is handled for the previous design (Fig. 1) that has one FSO channel with another design (Fig. 2) having two channels.

Figure 2 shows an enhancement of the system performance in dust storms condition and extends the link range by using two channels enforced with two optical amplifiers EDFAs. The new system design is consisted of two FSO channels with the fork power splitter and power combiner in order to take advantage of the constrictive combination of the light power, and an optical amplifier is used to increase the performance of the system. The gain of the amplifier is set to be $20 \mathrm{~dB}$. The amplified signal is then directly sent to the receiver through the free space optical channel, and another one is suited in the reversed side as shown in Fig. 3.

The parameters in Table 2 are used to simulate the channel transmission in the FSO systems via the optical simulator software, "Optisystem ${ }^{\mathrm{TM}}$ ".

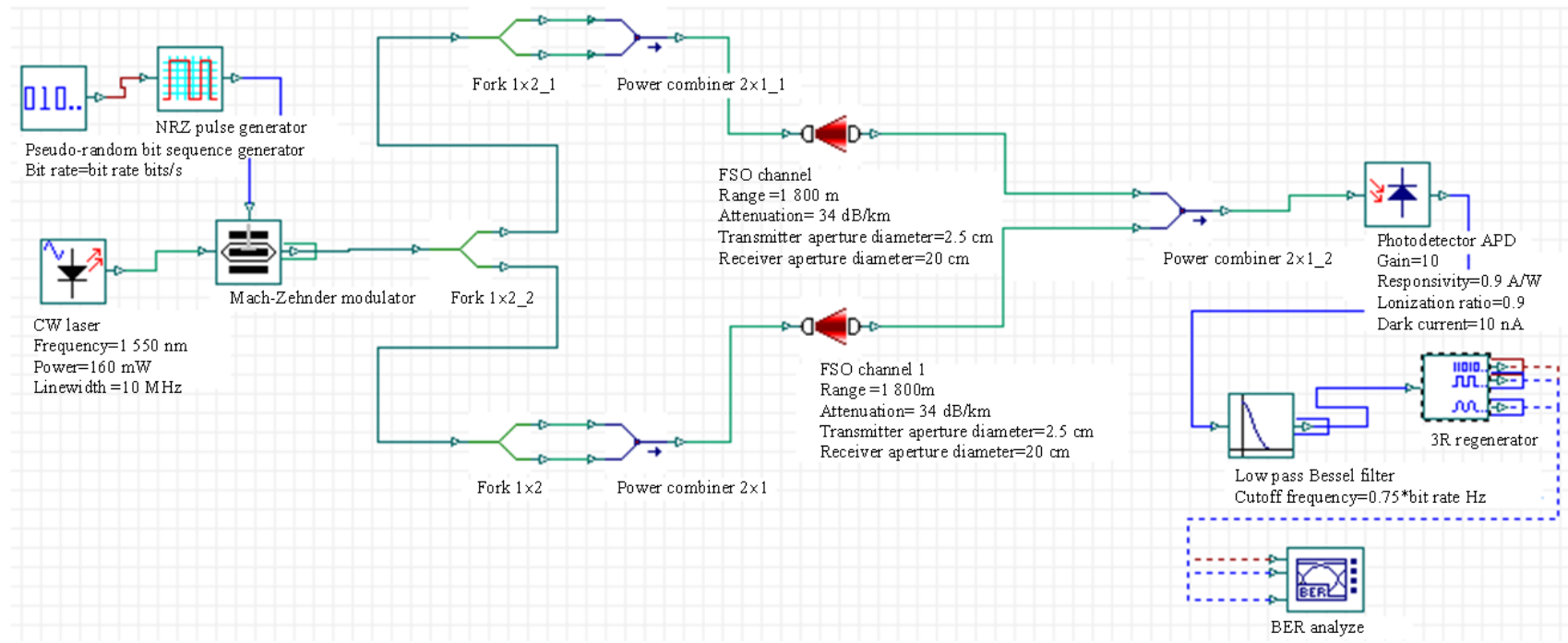

Fig. 2 Two-channel FSO system.

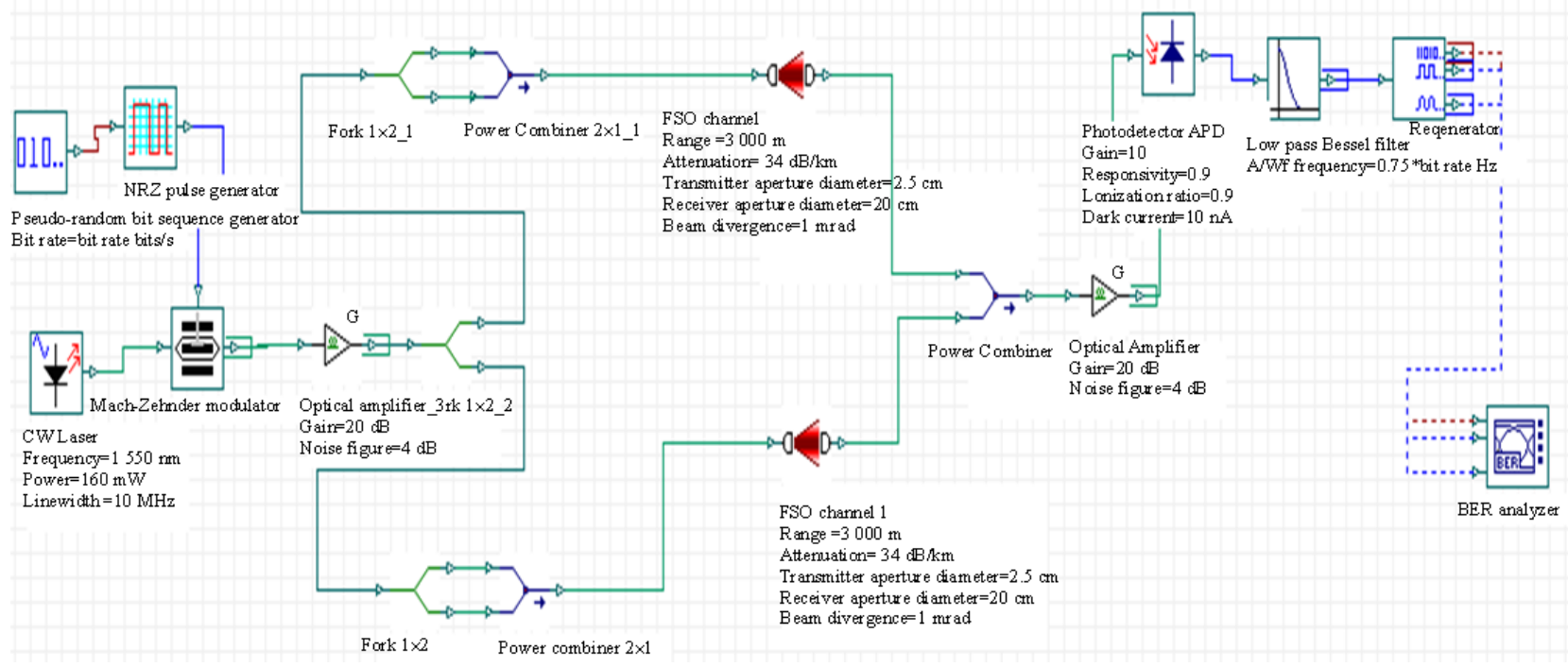

Fig. 3 New FSO system design to enhance the overall performance in complex weather conditions. 
Table 2 FSO link parameters.

\begin{tabular}{lc}
\hline \multicolumn{1}{c}{ Channel configurations } & Parameter \\
\hline Dynamic range & $3000 \mathrm{~m}$ \\
Attenuation for dust storms & $34 \mathrm{~dB} / \mathrm{km}-242 \mathrm{~dB} / \mathrm{km}$ \\
Diameter of the aperture of the receiver & $20 \mathrm{~cm}$ \\
Diameter of the aperture of the transmitter & $2.5 \mathrm{~cm}$ \\
Transmitter and receive loss & $1 \mathrm{~dB}$ \\
Transmission power & $160 \mathrm{mV}$ \\
Wavelength & $1550 \mathrm{~nm}$ \\
Beam divergences & $1 \mathrm{mrad}$ \\
\hline
\end{tabular}

\section{Results and discussion}

In this section, numerical results for the BER performance of FSO links based on the above system model will be presented for various number links designs with different wavelengths and distances. The FSO system is considered with transmitting and receiving apertures with the size of $2.5 \mathrm{~cm}$ and $20 \mathrm{~cm}$, respectively. The attenuations are measured with different attenuations ranges, according to the Kim model, which is illustrated at $1550 \mathrm{~nm}$ as shown in Fig. 4 and at $850 \mathrm{~nm}$ as shown in Fig. 5. It is obvious that using multiple channels with EDFA amplifiers at $1550 \mathrm{~nm}$ could have the best result for different attenuations. Figure 6 shows the system's quality factor while Fig. 7 shows the BER comparison among one channel, two channels, and two channels with EDFA under the moderate dust scenario.

The BER performance is illustrated in Fig. 7, in which the systems with one and two channels in general are affected by the weather attenuation from $1 \mathrm{~km}$ and BER becomes approximately $1.8 \mathrm{E}-13$ at the distance of $1250 \mathrm{~m}$.

Figure 8 illustrates the eye diagram distortion utilizing the BER analyzer in Optisystem ${ }^{\mathrm{TM}}$. The enhancement in the bit error rate is clearly shown by using the new design of two channels with EDFAs. The approximate BER is $2.12 \mathrm{E}-10$ at the distance of $2300 \mathrm{~m}$.

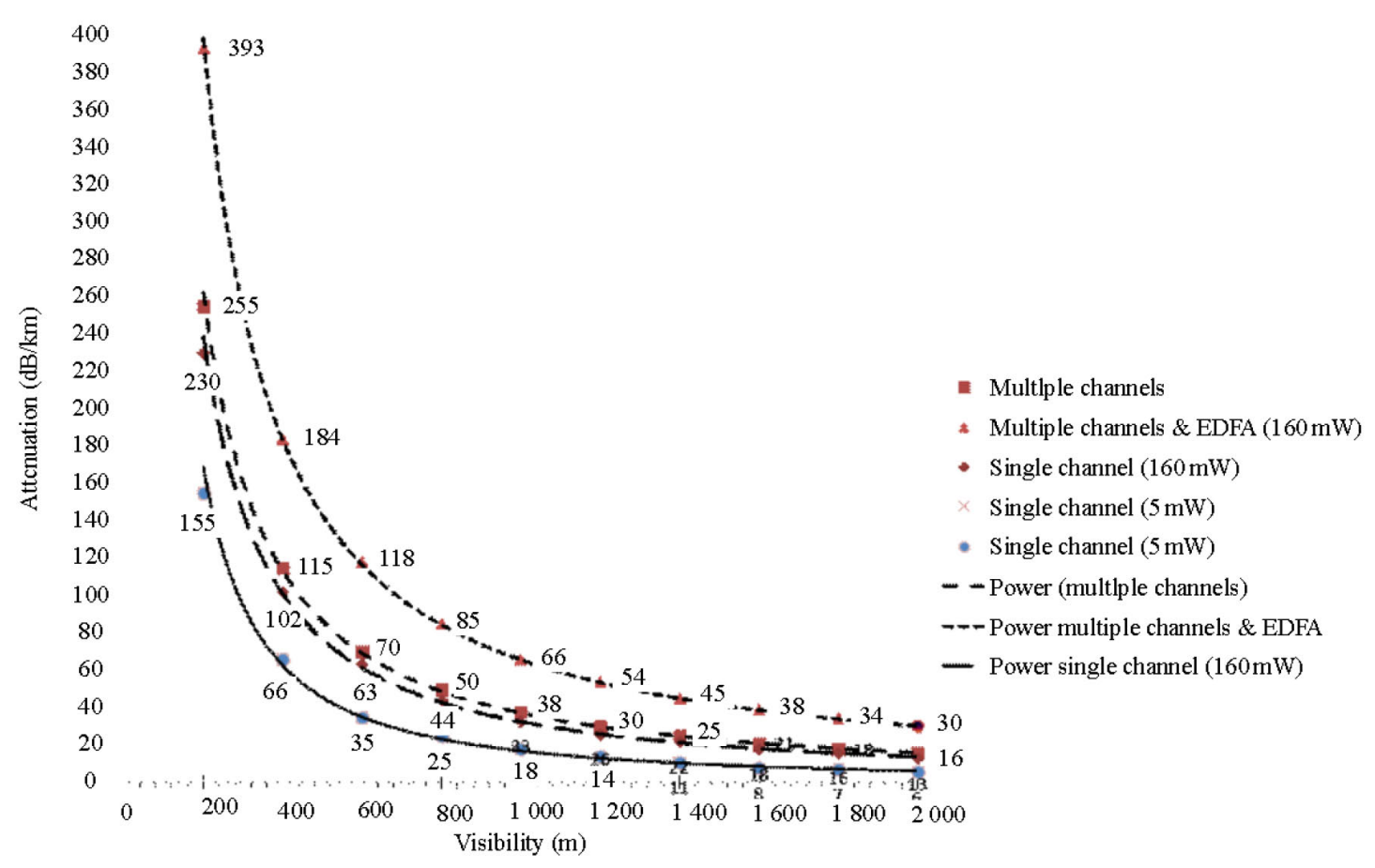

Fig. 4 Variation of the attenuation at $1550 \mathrm{~nm}$ in the function of visibility, compared, using the Kim model. 


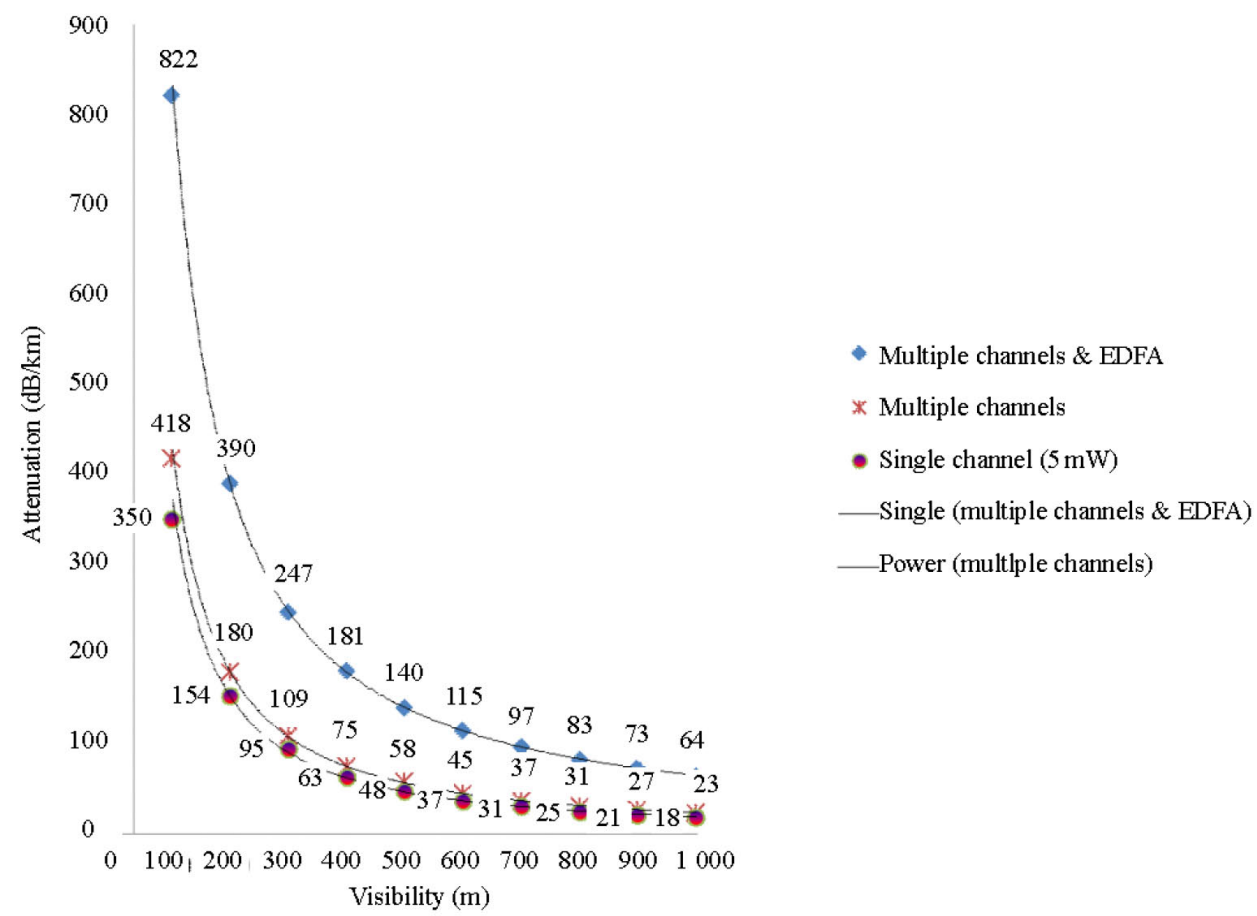

Fig. 5 Variation of the attenuation at $850 \mathrm{~nm}$ in the function of visibility, compared, using the Kim model.

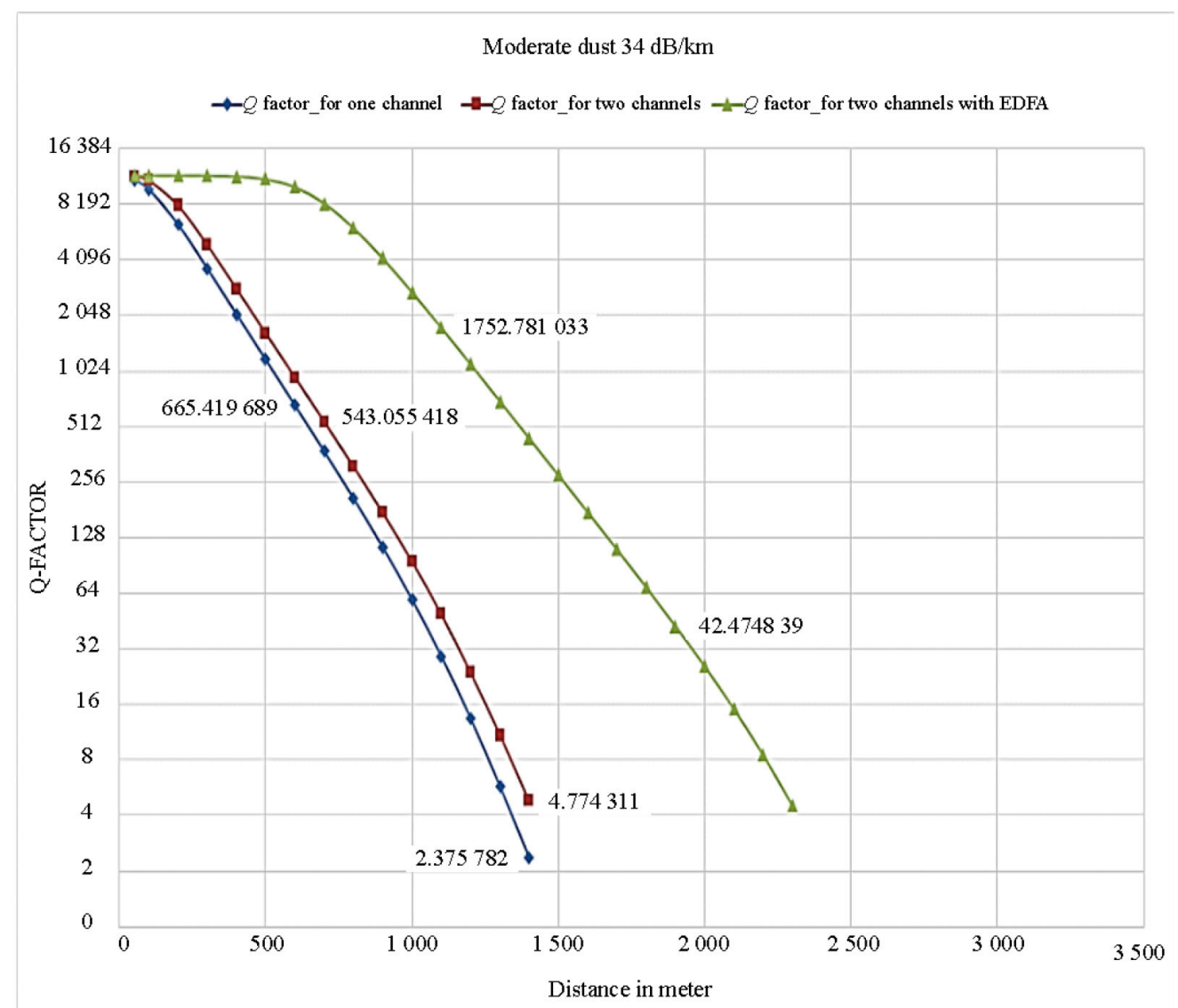

Fig. 6 Quality factor at $34 \mathrm{~dB} / \mathrm{km}$ according to the distance. 


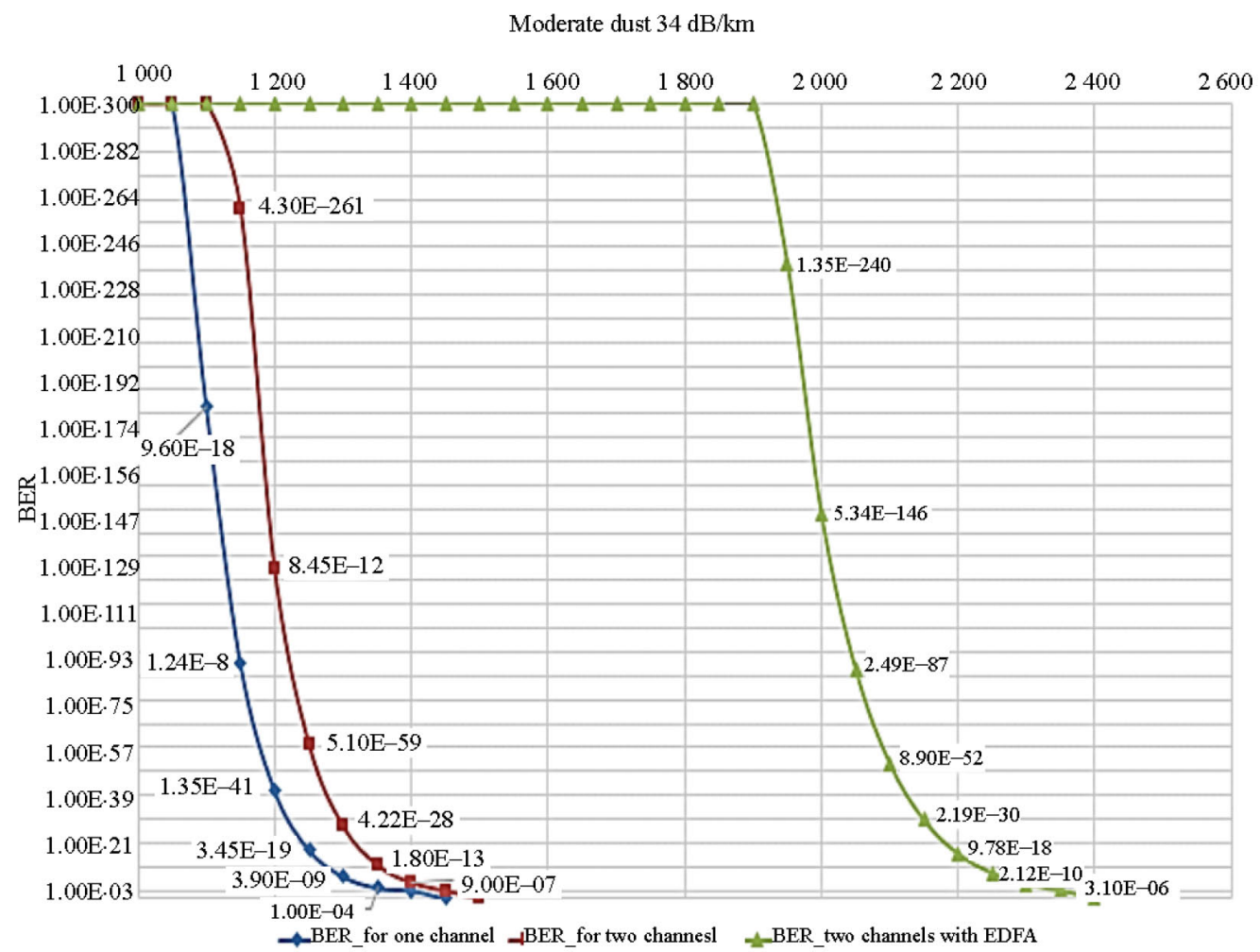

Fig. 7 BER performance comparison according to the distance at $34 \mathrm{~dB} / \mathrm{km}$.

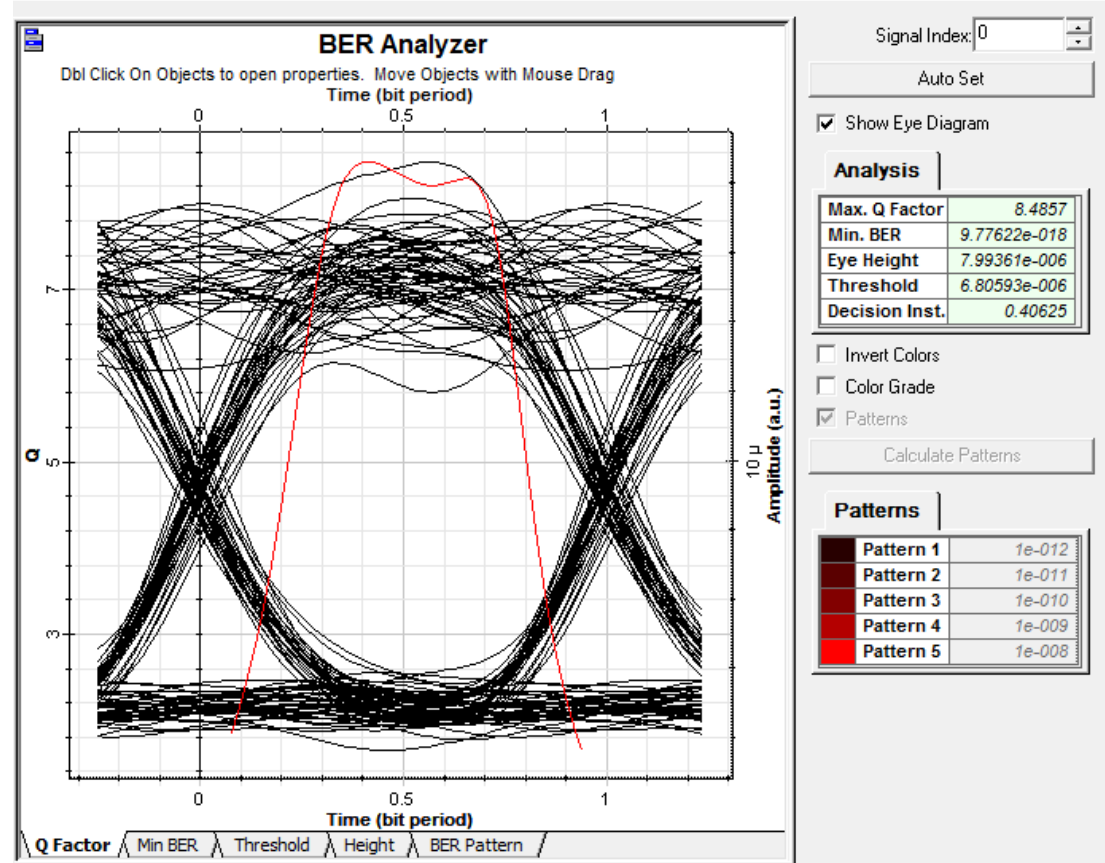

Fig. 8 Eye diagram showing BER performance on moderate dust $32 \mathrm{~dB} / \mathrm{km}$ of two channels and EDFA FSO system at $2.3 \mathrm{~km}$.

The heavy dust storms are considered as a design's challenge since its attenuation is calculated using the Kim model with a visibility of $70 \mathrm{~m}$ to be around $242 \mathrm{~dB} / \mathrm{km}$. Figure 9 shows the system's quality factor, and Fig. 10 shows the BER comparison among one channel, two channels, and two channels with EDFA under the heavy dust scenario. 


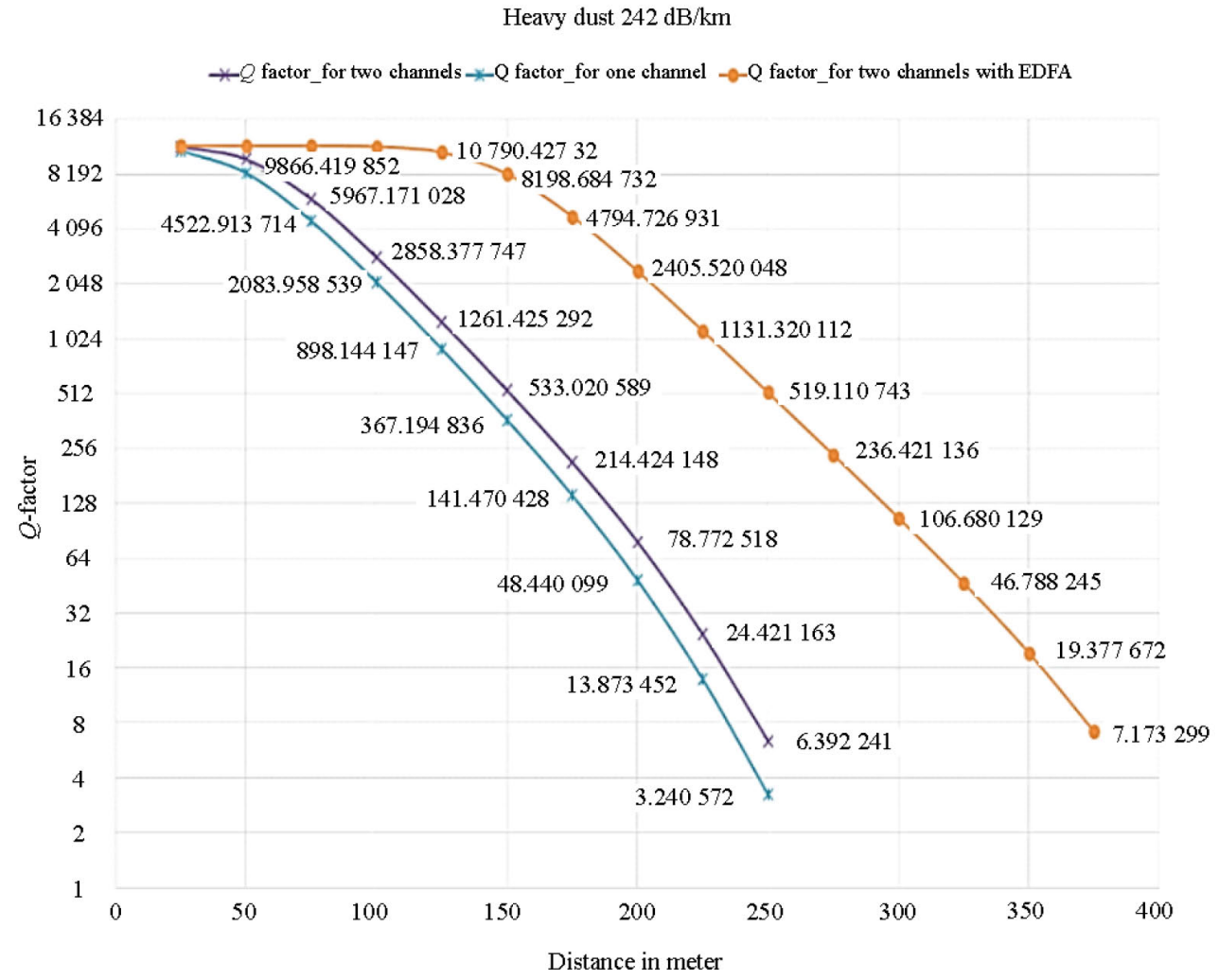

Fig. 9 Quality factor at $242 \mathrm{~dB} / \mathrm{km}$ according to the distance.

Moderate dust $242 \mathrm{~dB} / \mathrm{km}$

Distance in meter

180

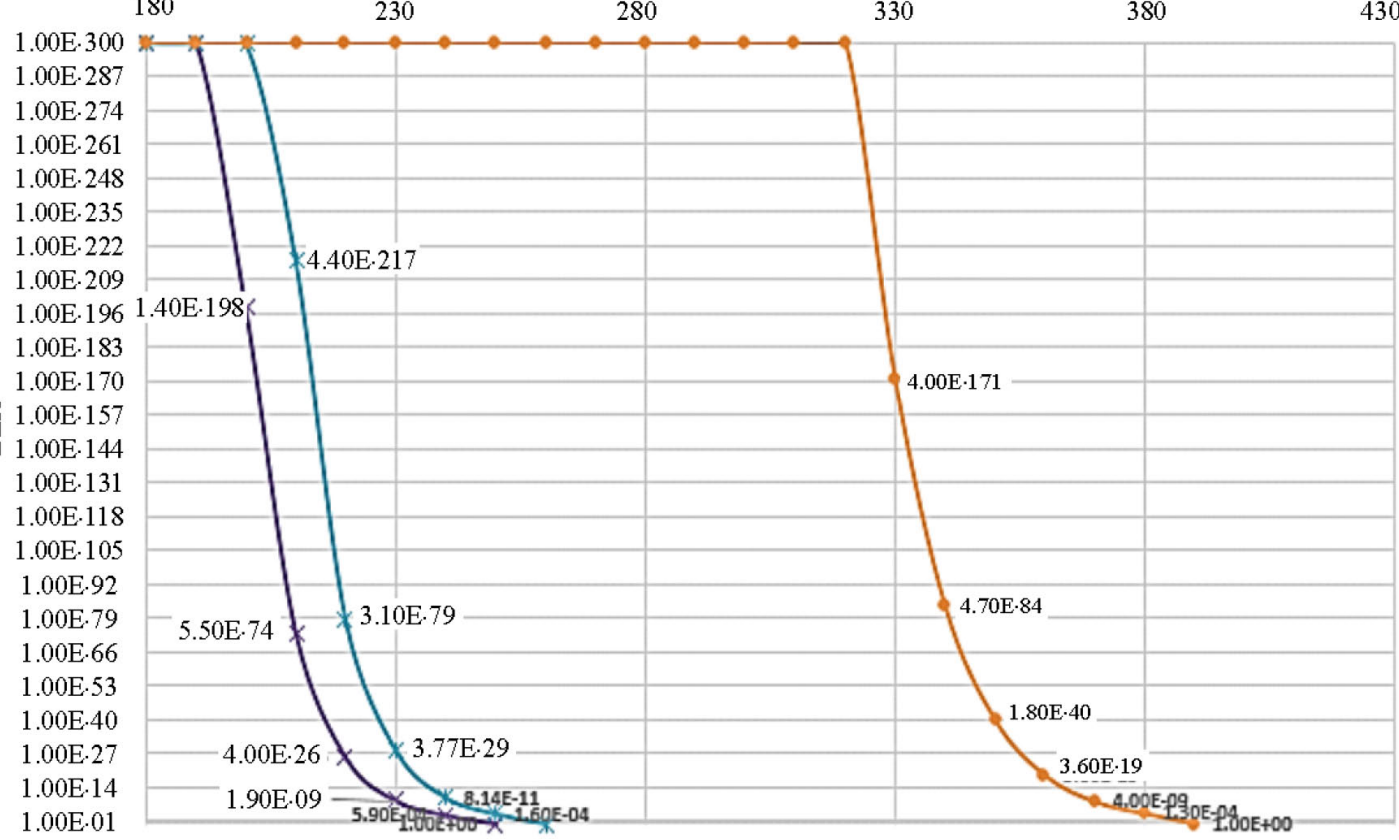

*BER_for one channel $\%$ BER_for two channel -BER_two channel with EDFA

Fig. 10 BER performance comparison according to the distance at $242 \mathrm{~dB} / \mathrm{km}$. 
The BER performance in Fig. 10 and the eye diagram in Fig. 11 clearly show that the performances are improved significantly with the increasing number of transmitting links, which in effect reduces the effect of the weather turbulence variance for the delivering channel, for example, the BER performance for $242 \mathrm{~dB} / \mathrm{km}$ is zero using the proposed design of two channels and EDFAs at the distance of $280 \mathrm{~m}$. In this point, the other designs are at the highest BER. Further, the EDFA amplifiers will also increase the channels performance as BER for $242 \mathrm{~dB} / \mathrm{km}$ at the distance up to 38 .

In distance $0 \mathrm{~m}$, all wavelengths have equivalent BER, knowing that the minimum acceptable BER is $1.0 \mathrm{E}-9[10]$.

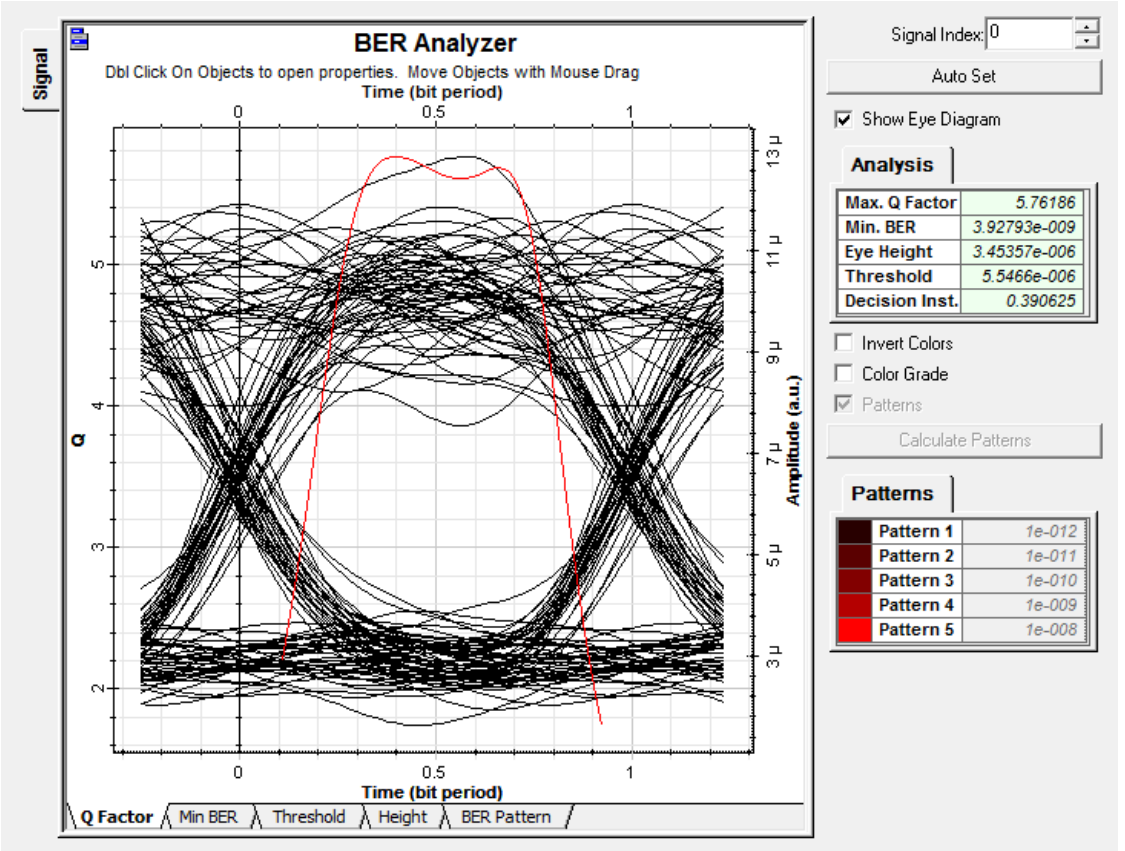

Fig. 11 Eye diagram showing BER performance on heavy dust $242 \mathrm{~dB} / \mathrm{km}$ of two channels with EDFA FSO system at $380 \mathrm{~m}$.

Although there is some discrepancy observed in these different designs, the performance will still be limited to some distance, however an increase in the number of links and the power of the amplifiers will increase the performance of the FSO system.

\section{Conclusions}

In this paper, the FSO system BER performance has been investigated over different turbulence atmospheric weathers. Results demonstrate that FSO links could be efficiently effective for higher attenuations such as in the heavy fog and dust by using multiple channels with EDFA amplifiers. Moreover, the effect of the attenuation will be decreased, and the BER performance will be increased via the rising number of transmitting links plus the power of amplifiers.

In addition, the performance of the links bounded by the wavelength that was observed, however the numerical result shows that at higher attenuation of different wavelengths the BER have the same performance. As a result, the BER performance could be significantly improved, and further communicating distance could be achieved at $1550 \mathrm{~nm}$ with multiple channels and EDFA amplifiers. Finally, this improvement in performance has led to enhance the quality of the service.

Open Access This article is distributed under the terms of the Creative Commons Attribution 4.0 International License (http://creativecommons.org/ licenses/by/4.0/), which permits unrestricted use, distribution, and reproduction in any medium, provided you give 
appropriate credit to the original author(s) and the source, provide a link to the Creative Commons license, and indicate if changes were made.

\section{References}

[1] I. I Kim, B. McArthur, and E. J. Korevaar, "Comparison of laser beam propagation at $785 \mathrm{~nm}$ and $1550 \mathrm{~nm}$ in fog and haze for optical wireless communications," SPIE, 2001, 4214(2): 26-37.

[2] A. Raja, Q. Kagalwala, T. Landolsi, and M. El-Tarhuni, "Free-space optics channel characterization under UAE weather conditions," in 2007 IEEE International Conference on Signal Processing and Communications, Dubai, pp. 856-859, 2007.

[3] J. Perez, Z. Ghassemlooy, S. Rajbhandari, M. Ijaz, and H. Minh, "Ethernet FSO communications link performance study under a controlled fog environment," IEEE Communications Letters, 2012, 16(3): 408-410.

[4] Z. Ghassemlooy, J. Perez, and E. Leitgeb, "On the performance of FSO communications links under sandstorm conditions," in International Conference on Telecommunications, 2013, pp. 53-58.

[5] M. Karimi and M. Nasiri-Kenari, "Free space optical communications via optical amplify-and-forward relaying," Journal Lightwave Technology, 2011,
29(2): 242-248

[6] H. A. Fadhil, A. Amphawan, H. A. B. Shamsuddin, T. H. Abd, H. M. R. Al-Khafaji, S. Aljunid, et al., "Optimization of free space optics parameters: an optimum solution for bad weather conditions," Optik - International Journal for Light and Electron Optics, 2013, 124(19): 3969-3973.

[7] N. A. Mohammed, A. S. El-Wakeel, and M. H. Aly, "Performance evaluation of FSO link under NRZ-RZ line codes, different weather conditions and receiver types in the presence of pointing errors," Open Electrical \& Electronic Engineering Journal, 2014, 6(1): 28-35.

[8] S. H. Alnajjar, M. S. R. F. Malek, and M. S. Ahmad, "Low-altitude platform to enhance communications reliability in disaster environments," Journal of Advances in Information Technology, 2014, 5(1): 21-30.

[9] Z. Hu, H. F. Chou, J. E Boors, and D. J. Blumenthal, "40-gb/s optical clock recovery using a compact traveling-wave electroabsorption modulator-based ring oscillator," IEEE Photonics Technology Letters, 2004, 16(5): 1376-1378.

[10] B. Wandernoth, "5 photon/bit low complexity $2 \mathrm{MBit} / \mathrm{s}$ PSK transmission breadboard experiment with homodyne receiver applying synchronization bits and convolutional coding," in 20th European Conference on Optical Communications, Firenze, pp. 59-62, 1994. 